\title{
Endoscopic sphincterotomy without cholecystectomy for gall stone pancreatitis
}

\author{
C R B Welbourn, D E Beckly, I A Eyre-Brook
}

\begin{abstract}
Fifty one patients (median age 78 , range 54-92) who had endoscopic sphincterotomy for gall stone pancreatitis without planned cholecystectomy were followed up to assess the effectiveness of this treatment in preventing further acute pancreatitis. In 48 patients sphincterotomy was technically successful and none had further acute pancreatitis after a mean follow up of $\mathbf{2 6 . 9}$ months. In three patients sphincterotomy was incomplete and two developed recurrent pancreatitis. These results suggest that endoscopic sphincterotomy alone is an acceptable alternative to cholecystectomy in the prevention of further attacks of acute pancreatitis in the elderly.

(Gut 1995; 37: 119-120)
\end{abstract}

Keywords: gall stone, pancreatitis, endoscopic sphincterotomy, cholecystectomy.

Acute gall stone pancreatitis is a potentially lethal disease in the elderly. Established treatment consists of supportive medical therapy followed by planned cholecystectomy. ${ }^{1}$ Removing the gall bladder effectively prevents further episodes of acute pancreatitis ${ }^{2}$ but in the elderly has considerable morbidity. ${ }^{3}$ If the gall bladder is left intact the risk of further pancreatitis is cumulative and at least $35 \% .^{2} 45$ In the elderly patient with gall stone jaundice endoscopic sphincterotomy (ES) has been established as definitive treatment if gall bladder symptoms are absent, ${ }^{67}$ and has the advantage of lower morbidity than cholecystectomy in these patients. Recent evidence suggests that early ES also reduces the morbidity of an attack of severe acute gall stone pancreatitis. ${ }^{8}$ The impact of ES on the risk of subsequent acute pancreatitis in the presence of confirmed gall bladder stones is, however, unknown. ${ }^{9}$ We studied whether ES without cholecystectomy prevents recurrent gall stone pancreatitis in the elderly patient with symptomless gall bladder stones.

\section{Methods}

Data were collected prospectively in two district general hospitals on all patients undergoing endoscopic retrograde cholangiography (ERC) without cholecystectomy for gall stone pancreatitis. All patients had intact gall bladders but no symptoms from gall bladder stones. They were selected for this treatment by the referring clinician if cholecystectomy was deemed undesirable because of advanced age or poor medical condition. Pancreatitis was diagnosed when a serum amylase concentration of over $1000 \mathrm{U} / \mathrm{l}$ (normal range 70-300 $\mathrm{U} / \mathrm{l})$ was present in a patient with an appropriate clinical presentation. Gall stone pancreatitis was established when gall stones were shown in the gall bladder or bile ducts by ultrasound scan or ERC. In each centre ES was performed by a single endoscopist and data were collected since the technique was introduced to the hospital (October 1983 in Plymouth; February 1989 in Taunton) until the end of the study in November 1993. ES was deemed complete when the entire submucosal portion of the bile duct was divided and all duct stones cleared. Bile ducts were cleared of stones by balloon or basket trawl and in three patients mechanical lithotripsy was necessary. Three patients had temporary biliary drainage (one pig tail stents, two nasobiliary drainage) after initial ERC before duct clearance was achieved at a second ERC. One patient had the permanent insertion of pig tail stents after incomplete extraction of multiple large duct stones. All patients were followed up by outpatient or telephone contact. Further details of symptoms, hospital admissions, and cause of death after ES were obtained from hospital or general practitioner records.

\section{Results}

Fifty one patients (median age 78 , range 54-92) were treated expectantly by two endoscopists without planned cholecystectomy (Figure). Thirty eight $(74.5 \%)$ were aged over 70. Two patients died within 30 days of ES, one (age 63) of bronchopneumonia and cirrhosis, the other (age 86) of bronchopneumonia. Two patients were admitted to a high dependency unit because of oversedation and recovered completely within 24 hours. There were no episodes of acute pancreatitis, no overt gastrointestinal haemorrhage, and no duodenal perforations after ERC.

Forty eight patients had a technically complete ES and required 57 ERCs to achieve duct clearance (mean 1.19). Thirty eight patients had a median of two bile duct stones (range 1-15), which were of median size $7 \mathrm{~mm}$ (range $1-15)$. No duct stone was found in the remaining 13 patients. Seven patients had a second ERC because of failure to clear the bile duct after a median two weeks (range 1-8) and in two patients ERC was repeated routinely to check the bile duct was clear. Gall bladder stones were shown to be present at the time of ERC in 43 of $48(90 \%)$ patients with successful ES. In the remaining five patients the gall bladder did not fill at ERC. Forty six of 48 


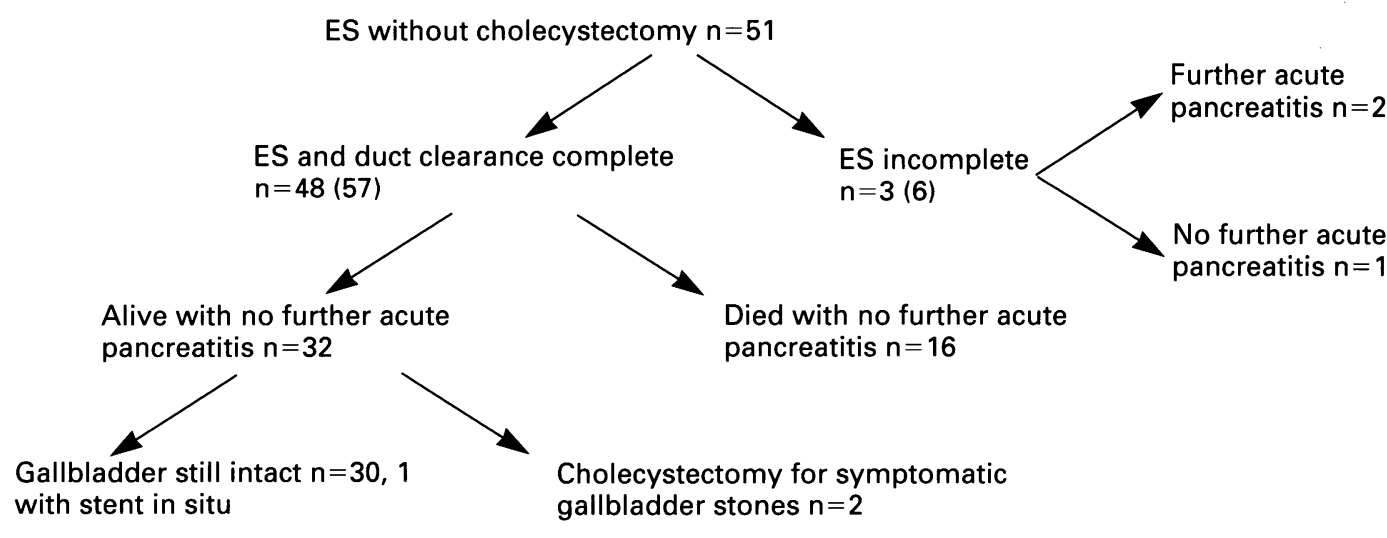

Results of treatment of gall stone pancreatitis by ES (endoscopic sphincterotomy). The number of ERC procedures in each group is shown in brackets.

patients with technically successful ES survived longer than one month, of whom 14 died from non-biliary causes a mean of 25.0 months after ES (range 2-94). None of these patients had further pancreatitis. The remaining 32 surviving patients had no further pancreatitis at a mean 29.4 months (range 2-121), a total of 108 patient years of pancreatitis free survival. There was no correlation between age at ES and pancreatitis free survival. Four of these patients had biliary colic or cholecystitis during follow up, of whom two underwent cholecystectomy after a mean 13.5 months.

In three patients ES was recorded in the endoscopic record as inadequate with incomplete incision of the submucosal bile duct. These failures resulted from an overhanging fold of mucosa obscuring the bile duct $(n=1)$, technical difficulty with a broken scope $(n=1)$, and patient intolerance of the procedure $(n=1)$. The first two of these patients developed a further attack of acute pancreatitis on follow up. Of these, the first patient's bile duct was cleared of stones during incomplete ES and residual stones were shown in the gall bladder; and the second had a single $2 \mathrm{~mm}$ stone left in the bile duct and was treated expectantly.

\section{Discussion}

In this series no further episode of acute pancreatitis occurred during 108 patient years of follow up after successful ES. It is known that ES can reduce the morbidity and mortality of severe acute gall stone pancreatitis. ${ }^{7}$ These results suggest that ES may also prevent further acute pancreatitis if the bile duct is cleared but confirmed stones remain in the gall bladder, thus extending the findings of others. ${ }^{10}$ Recurrent pancreatitis was also prevented in the one patient who had permanent insertion of pig tail stents after incomplete duct clearance.

Stones were present in $74.5 \%$ of bile ducts and were of median size $7 \mathrm{~mm}$. This may overestimate the size of duct stones that cause pancreatitis. In 13 patients the ducts were clear of stones, in keeping with the suggestion that it is small stones that cause pancreatitis and then pass spontaneously. ${ }^{111} \mathrm{We}$ presume that ES permits any further stones in the bile duct to pass into the duodenum without causing recurrent pancreatitis. The finding that recurrent pancreatitis occurred in one patient with a clear bile duct and remaining gall bladder stones, but with an incomplete ES, supports this.

Studies of ES without cholecystectomy in gall stone jaundice suggest that less than $10 \%$ of patients will develop gall bladder symptoms requiring cholecystectomy within two years. ${ }^{6}$ This study suggests the risk after acute gall stone pancreatitis may be similar with only $4 \%$ requiring cholecystectomy after a mean follow up of nearly 2.5 years. As gall stone pancreatitis increasingly affects patients over $70^{12}$ these results suggest that in these patients ES might substitute for cholecystectomy. If a policy of ES without cholecystectomy were to be adopted in the elderly and unfit the risk of recurrent attacks of pancreatitis would be minimised and many patients would be spared the discomfort and potential complications of cholecystectomy.

An abstract relating to this work was presented to the British Society of Gastroenterology, 23-25 March 1994 and appeared in abstract form in Gut 1994; 35 (suppl 2): S57.

1 Carter DC. Gallstone pancreatitis. In: Carter DC, Warshaw AL, eds. Pancreatitis. Edinburgh: Churchill Livingstone, 1989: $58-70$.

2 Paloyan D, Simonowitz D, Skinner DB. The timing of biliary tract operations in patients with pancreatitis associ-
ated with gallstones. Surg Gynecol Obstet 1975; 141: ated with

3 Irvin TT, Arnstein PM. Management of symptomatic gallstones in the elderly. Br $\mathcal{F}$ Surg 1988; 75: 1163-5.

4 Elfström J. The timing of cholecystectomy in patients with gallstone pancreatitis. A retrospective analysis of 89 patients. Acta Chir Scand 1989; 144: 487-90.

5 Burch JM, Feliciano DV, Mattox KL, Jordan GL. Gallstone pancreatitis. The question of time. Arch Surg 1990; 125: 853-9.

6 Neoptolemos JP, Carr-Locke DL, Fraser I, Fossard DP. The management of common bile duct calculi by endoscopic sphincterotomy in patients with gallbladders in scopic sphincterotomy in patient

7 Martin DF, Tweedle DEF. Endoscopic management of common duct stones without cholecystectomy. Br $\mathfrak{F}$ Surg 1987; 74: 209-11.

8 Fan S-T, Lai ECS, Mok FPT, Lo C-M, Zheng S-S, Wong J. Early treatment of acute biliary pancreatitis by endoscopic papillotomy. $N$ Engl $\mathcal{f}$ Med 1993; 328: 228-32.

9 Neoptolemos JP. Endoscopic sphincterotomy in acute gallstone pancreatitis. Br $\mathcal{F}$ Surg 1993; 80: 547-9.

0 Hill J, Martin DF, Tweedle DEF. Risks of leaving the gallbladder in situ after endoscopic sphincterotomy for bile duct stones. Br f Surg 1992; 78: 554-7.

11 Johnson AG, Hosking SW. Appraisal of the management of bile duct stones. Br f Surg 1987; 74: 555-60.

12 Corfield AP, Cooper MJ, Williamson RCN. Acute pancreatitis: a lethal disease of increasing incidence. Gut 1985; 26: 724-9. 